Original Research Paper

\title{
Multi-Criteria Trust Establishment for Multi-Agent Systems Based on Fuzzy Logic
}

\author{
${ }^{1}$ Abdullah Aref and ${ }^{2}$ Eman Omar \\ ${ }^{1}$ Department of Computer Science, King Hussein School of Computing Sciences, \\ Princess Sumaya University for Technology, Amman, 11941, Jordan \\ ${ }^{2}$ Department of Computer Science, University of the People, USA
}

\section{Article history}

Received: $15-07-2020$

Revised: 02-11-2020

Accepted: 03-11-2020

Corresponding Author:

Abdullah Aref

Department of Computer

Science, King Hussein School

of Computing Sciences,

Princess Sumaya University for

Technology, Amman, 11941,

Jordan

Email: a.aref@psut.edu.jo

\begin{abstract}
Multi-Agent Systems (MASs) are increasingly accepted for modeling virtual complex distributed systems, such as virtual societies and smart grids, due to agents' autonomy, flexibility and pro-activity. As autonomous, goal-driven agents can mislead others intentionally or accidentally by inaccurately reporting their competencies and abilities, the use of trust modeling deemed essential for successful interactions in MASs. The concept of trust is complex, multidimensional and includes more than just evaluating how honest interaction partners are. This study describes an explicit, multi-criteria, trust establishment model based on fuzzy logic to guide trustees in MASs to improve their level of trust as perceived by the trustor by tuning up their behaviors to attract more interactions with potential partners. When trustors are willing to provide feedback for interactions in the form of a single satisfaction value per multi-criterion interaction, the model attempts to predict the necessary improvement per criterion. We evaluated the performance of the proposed model using simulation. The results indicate that the model can help trustees achieve higher trust levels and get better chances to be selected as partners for interactions in MASs when trustors select trustees based on trust.
\end{abstract}

Keywords: Multi-Agent Systems, Trust Establishment, Fuzzy Logic

\section{Introduction}

Multi-Agent Systems (MASs) are increasingly accepted for modeling virtual complex systems such as e-commerce (Ramchurn et al., 2004) and smart grids (Moradi et al., 2016), where agents can come from various backgrounds with diverse capabilities, agents can join or leave the system and individual agents independently make decisions (Yu et al., 2013b). As individual agents' capabilities in a MAS can be limited, individual agents may need to collaborate with other agents to accomplish their goals (Yu et al., 2013b). As autonomous agents in MASs may have their own costume set of beliefs and may make inaccurate statements regarding their abilities and competencies, there is a need for using effective trust assessment models to help trustors maximize interactions' benefits (Burnett, 2011). Unlike security techniques concerned with ensuring data integrity and data confidentiality, trust evaluation models offer a way to protect trustors from malicious trustees (Yu, 2014).
When agents' interactions in a MAS are based on trust, trust establishment schemes can help direct trustees ascertain greater trust levels and possess more significant impacts on interactions' outcomes.

Unfortunately, the term "trust establishment" was used in different manners in trust management literature. Few papers in the literature use the term "trust establishment" to help trustees be more trustworthy (Sen, 2013; Tran et al., 2014; Aref and Tran, 2017b). In the domain of ad-hoc networks, the term refers to the trust evaluation of trustees from trustors' perspective, such as the work presented in (Saini and Gautam, 2011). In the domain of service-oriented computing, the term used to refer to bootstrapping trust (Malik and Bouguettaya, 2009).

Due to the simplicity of Fuzzy Logic Systems (FLSs) and their similarity to human reasoning, FLSs have been successfully used with various application areas (Georgoulas et al., 2012) including web services trust evaluation (Shirgahi et al., 2017), cloud computing trust evaluation (Supriya et al., 2016), social networks trust 
evaluation for (Singh and Sidhu, 2016a) and trust evaluation for multi-agent systems (Aref and Tran, 2018; 2014; Singh and Sidhu, 2016b; Shen et al., 2015).

This study describes a trust establishment model for multi-criteria services using fuzzy logic. Based on the reported level of satisfaction per criterion from trustors, the model directs trustees to improve their performance to become more trustworthy from the perspective of trustors.

To analyze the performance of the proposed model, we simulated the use of the model in various scenarios of trustors' levels of demands and levels of activity compared to the most relevant trust establishment models in the literature. The simulation analysis results indicate that the proposed model can help trustees become more trustworthy compared to the model that uses explicit feedback and does not use fuzzy logic, at a comparable cost in terms of provided utility gain, especially for demanding trustors.

The paper is organized as follows:

An overview of fuzzy logic is presented next in section 2 , before overviewing related work in section 3. Section 4 presents a system overview, before detailing of the proposed model in section 5. The details of the performance evaluation is described in section 6 , followed our conclusions and ideas for future work.

\section{Background}

\section{Fuzzy Logic}

Fuzzy Logic provides a non linear mapping of input data vector and scalar output (Mendel, 1995) where human knowledge can be presented as a set of basic rules in linguistic terms that maps the input into the corresponding output (Ross, 2010). A typical FLS contains an inference engine, a set of fuzzy logic rules, a fuzzifier and defuzzifier (Mendel, 1995). Figure 1 demonstrates the basic architecture of a typical FLS. Various extensions to the basic architecture have been proposed in the literature such as the hierarchical extensions described in (Raju et al., 1991).

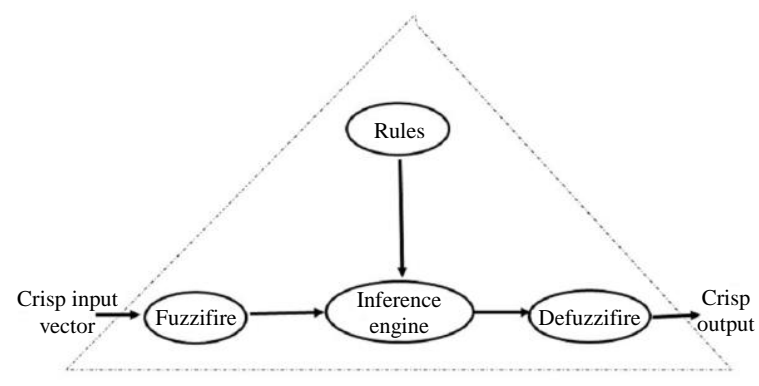

Fig. 1: Fuzzy logic system
FLSs enable transitions from one input state to another in a smooth manner (Muñoz et al., 2014) as fuzzy sets implicate progressive membership of elements in sets. The concept of Membership Function (MF) determines the level to which a fuzzy variable is a member of a set where zero represents one extreme one represents the other extreme (Griffiths et al., 2006).

\section{Related Work}

Various models for trust evaluation have been proposed in the literature of trust management for MASs (Yu et al., 2013b; Pinyol and Sabater-Mir, 2013; Granatyr et al., 2015), which we consider as not directly related to models of trust establishment. On the other hand, little work contributed to modeling the trust establishment, which is the main objective of this study.

Initial steps toward building trust establishment models can be found in (Tran and Cohen, 2004; Zhang and Cohen, 2007).

The former one attempts to model the needs of buying agents in the e-marketplace, whereas the second one uses trust-gain to motivate honesty in the emarketplace.

Social Welfare Optimizing Reputation-aware Decision making (SWORD) model proposed (Yu et al., 2013c) for situations when a trustee can effectively fulfill a limited number of requests per unit time. Beyond that limit, the performance of the trustee may be negatively impacted as well as its reputation. SWORD acts as a mediator between trustees and trustors to balance the workload between reliable trustees, taking into account trustworthiness differences.

Centralized trust establishment models, such as SWORD, may work well for application with centralized nature, such as online crowd-sourcing. However, the centralized architecture conflicts with dynamic environments such as MASs environments, where agents can join and leave dynamically. As with trust evaluation models, centralized trust establishment models, such as SWORD, can suffer scalability issues. A distributed variant of SWORD is presented (Yu et al., 2013a) and named as the Distributed Request Acceptance approach for Fair utilization of Trustees (DRAFT). DRAFT aimed to help trustees with limited resources to dynamically decide if further requests for interaction can be accepted to maximize potential rewards without degrading the performance experienced by trustors. Aiming to prevent the trustworthiness score decline due to factors beyond the control of trustees.

Using Reputational Incentive (RI) model, each trustee builds its own set of models for trustors, continuously monitors its reputations to maintain an 
effective operation (Burnett et al., 2011). The model is a generic, decentralized model that can be used for a wide range of applications including those in dynamic environments such as MASs. The model uses accumulated reputation to adjust the performance of trustees and services assumed to be single criterion, or can be treated as such. Despite allowing multiple contexts, the model does not support checking and mapping context diversity. Furthermore, the model assumes that trustees can obtain their reputation precisely and does defend against potential attacks on reputation.

A distributed trust establishment model for ecommerce applications is presented (Tran et al., 2014) where each trustee builds its own set of models for trustors. The model uses Reinforcement Learning (RL) to classify trustors according to price and quality needs. The model assumes that trustors are cooperative and willing to provide precise explicit feedback and attempts to help trustees address trustors' needs based on trustors explicit feedback. As the model does not use third-party information, buyers are not motivated to lie. No further explicit defence against misleading information taking into account.

A set of decentralized trust establishment models using implicit feedback presented in the literature for situations where trustors are malicious or not willing to cooperate. Retention of trustors to interact is used as the single criterion, implicit feedback in the trust establishment model presented (Aref and Tran, 2015b). The model is refereed to as Reinforcement Learning based Trust Establishment (RLTE) and assumes that trustors initially have neutral demands. Trustees dynamically tune their performance using reinforcement learning depending on the retention of trustors. Similar to RLTE, other decentralized models for trust establishment presented in (Aref and Tran, 2016; 2015a; 2017a) use retention of trustors. Those models typically use different computation engines; (Aref and Tran, 2015a) uses fuzzy logic while (Aref and Tran, 2016) relies on a set of custom equations to help trustees dynamically predict trustors' behaviour.

The multi-criteria trust establishment model presented in (Aref and Tran, 2017a) attempts to predict both the proper value per criteria and its importance. The model tunes the performance of trustees with respect to each criterion based on the retention of trustors as well as the relative weight of the particular criterion. The model increases the predicted relative weight of a particular criterion if the corresponding trustor found to highly values that criterion. Otherwise, the model decreases the predicted relative weight of the criterion is decreased.
Aref and Tran (2017b) presented a Multi-Criteria Trust Establishment (MCTE) model. The model assumes that trustors are honest, cooperative and willing to provide a single aggregated satisfaction level regarding each transaction, but trustors do not provided the importance or level of satisfaction per criterion. MCTE used reinforcement learning to update the utility gain provided by trustees. The use of multi-criteria enables checking various contexts and the use of demands and weights can help in context mapping.

Recently a distributed, multi-criteria trust establishment model that integrates explicit and implicate feedback presented in (Aref and Tran, 2020b). The model, known as Integrated Trust Establishment (ITE), assumes that trustors are willing to provide a single aggregated satisfaction level regarding each transaction, but trustors do not provided the importance or level of satisfaction per criterion. ITE combine and implicit feedback from trustors in terms of their retention and explicit feedback regarding their satisfaction level with recent transactions. ITE adjust trustees behaviour according to the predicted importance of different service dimensions per trustor. As with MCTE, the use of multi-criteria enables checking various contexts.

In this study, we aim to evaluate fuzzy logic based on explicit feedback when it is available, rather than using reinforcement learning as in (Aref and Tran, 2017b; 2020b).

\section{System Overview}

Before detailing our proposed model, it may be necessary to present our assumptions and common notations that will be used later in the paper.

\section{Agent Architecture}

The MAS assumed to be composed of multiple agents, each of which assumed to have a set of modules, one of them is a Trust Management Module (TMM). Trust models of other agent are stored in the TMM which also, interfaces with other modules such as the module of communication (Sen, 2013). The TMM is assumed to include a component for trust evaluation and another one for trust establishment. The trust evaluation component should be responsible for modelling trustees form the perspective of trustors and help trustors select the an appropriate trustee to interact with. The trust establishment component should be responsible for modelling trustors from the perspective of trustees and help trustees taking actions to enhance their levels of trustworthiness. A general architecture for agent with a TMM is presented in Fig. 2. 


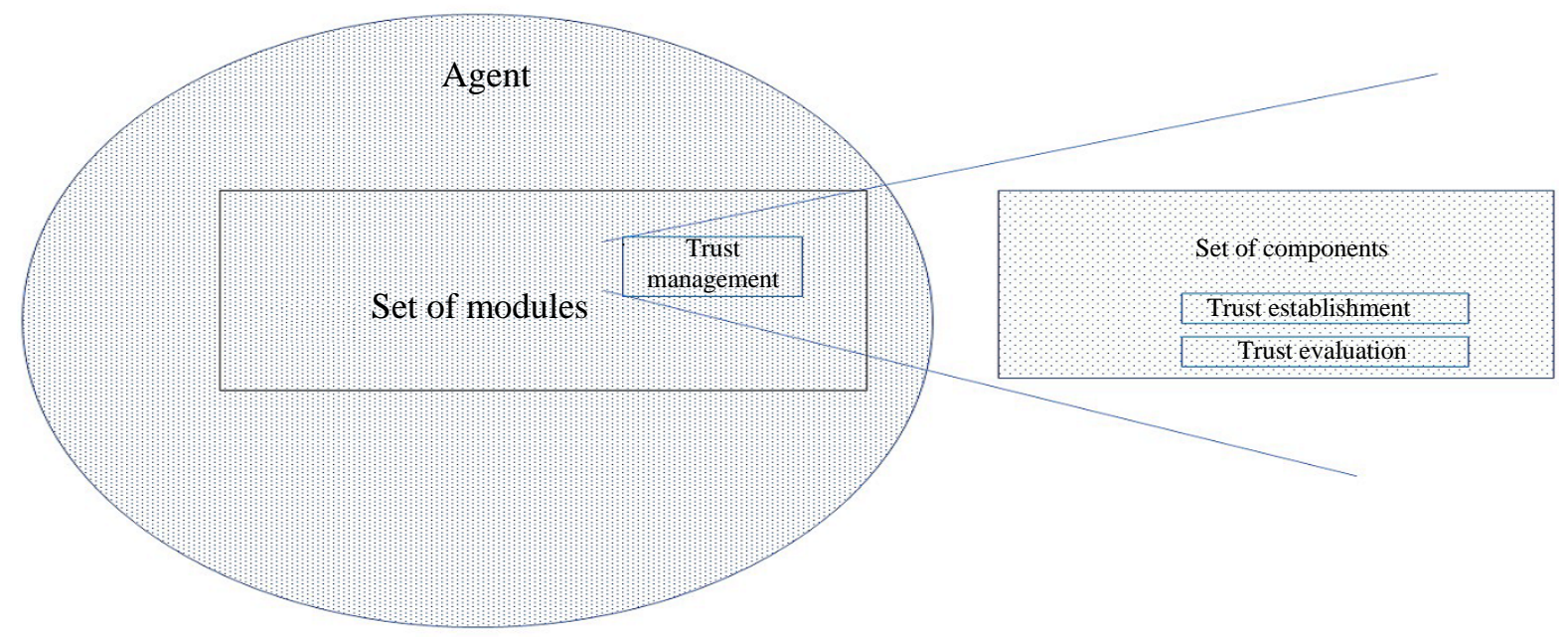

Fig. 2: General agent architecture with trust management module

\section{Agents and Tasks}

Each MAS is assumed to be composed of a set of n, possibly heterogeneous, agents $X=\left\{x_{1}, x_{2}, \ldots, x_{n}\right\}$. Agents in a MAS are interconnected, independent, autonomous and sufficiently intelligent to take actions and, if necessary, interact directly with each other. The set of trustors in a MAS can be denoted as $A=\left\{a_{1}, \ldots, a_{p}\right\}$ and the set of trustees can be denoted as $B=\left\{b_{1}, \ldots, b_{q}\right\}$ such that $A \cup B \subseteq X$. The set of possible tasks in the system can be denoted as $T=\left\{t_{1}, \ldots, t_{k}\right\}$, such that each task in the set has a set of dimensions (also known as criterion or features). We will denote the set of dimensions as $D=\left\{d_{1}, d_{2}, \ldots d_{m}\right\}$. Each trustees can carry out any number of tasks zero or more time. Possible tasks in $T$ may vary depending of the application area and levels of importance and demand for each dimension varies between trustors.

A trustor $a \in A$ that wants to have a task $t \in T$ realized, may depend on a trustee $b \in B$ to realize the task on its behalf.

Each task can be realized within a unit of time. However the time unit may vary depending on the application. In response to a particular request $r$ by $a$ to realize $t, b$ proposes to deliver a utility gain $U$ for $a$ as follows:

$$
U_{a}^{b}(t, r)=\sum_{i=1}^{m} \operatorname{Improvemet}_{a}^{b}\left(t_{d_{i}}, r\right)+\operatorname{Min}^{b}\left(t_{d_{i}}\right)
$$

- $U_{a}^{b}(t, r)$ is the overall utility gain trustee $b$ propose to trustor $a$ for request $r$ to fulfill $t$

- $\operatorname{MaxU}^{b}\left(t_{d_{i}}\right)$ and $\operatorname{MinU}^{b}\left(t_{d_{i}}\right)$ are the maximum and minimum possible values of utility gain that $b$ can deliver to dimension $d_{i}$ of task $t$
- Improvemet $t_{a}^{b}\left(t_{d_{i}}, r\right)$ is the amount of improvement trustee $b$ calculates for dimension $d_{i}$ of task $t$ in response to $s$ 's request $r$. Where:

$$
\begin{aligned}
& 0 \leq \text { Improvemet }_{a}^{b}\left(t_{d_{i}}, r\right) \\
& \leq\left(\operatorname{Max}^{b}\left(t_{d_{i}}\right)-\operatorname{MinU}^{b}\left(t_{d_{i}}\right)\right)
\end{aligned}
$$

The satisfaction result of each interaction is a real number in $[0,1]$ representing the Satisfaction's percentage (SAT) of trustor $a$ for task $t$ such that:

$\operatorname{SAT}_{a}^{b}(t, \operatorname{tra})=\sum_{i=1}^{m} \frac{w_{a}\left(t_{d_{i}}\right) * u_{a}^{b}\left(t_{d_{i}}, \operatorname{tra}\right)}{d_{a}\left(t_{d_{i}}\right)}$

Where:

$S A T_{a}^{b}(t, \operatorname{tra})=$ Trustor's $a$ satisfaction percentage of interaction tra with $b$ for task $t$

$w_{a}\left(t_{d_{i}}\right)=x$ 's importance or weight of dimension $d_{i}$ of task $t$. This weigh is not interaction dependent and does not vary with time for the same agent

$u_{a}^{b}\left(t_{d_{i}}\right.$, tra $)=$ The utility gain provided by $b$ to $a$ for dimension $d_{i}$ of task $t$ in the interaction tra, which can be interaction dependent

The fulfillment of a trustor's needs by a particular interaction is denoted as the percentage of satisfaction.

The average of satisfaction over various interactions is the direct trust evaluation of a trustee $b$ from the perspective of a particular trustor $a$ is the average 
satisfaction among various interactions between $a$ and $b$. The use of weight or importance, in Equation (3) enable varying interpretations of utility gain among agents. A wide range of trust evaluation models have been proposed in the literature based on fulfilling trustors' needs such as (Josang and Ismail, 2002; Huynh et al., 2006). Due to the distributed nature of MASs, we cannot assume the assistance of any central authority or entity to support trust-related communications.

\section{Trust Evaluation}

As the analysis of trust evaluation models is outside the scope of this study, we assume that each trustor has a trust evaluation model, Different sources of trust related information can be used to build such models.

We assume that trustors select trustees to interact with based on the utility gain expected from the interaction, which is calculated as:

$$
E V(y)=\operatorname{trust}_{b}^{a} * U_{a}^{b}
$$

Where:

$E V(y) \quad=$ The expected utility gain value

$U_{a}^{b} \quad=$ The utility gain proposed by trustee $b$

trust $_{b}^{a}(t)=$ Trust evaluation of $a$ for $b$

\section{A Multi-Criteria Trust Establishment Model using Fuzzy Logic (MCFTE)}

A trustor $a \in A$ that wants to have a task $t \in T$ realized, may depend on a trustee $b \in B$ to realize the task on its behalf.

In response to a particular request $r$ by $a$ to realize $t, b$ proposes to provide a utility gain $U$ for $a$ as calculated by Equation 1, section 4. The proposed model calculates the utility gain improvement per criterion using fuzzy logic based on explicit feedback from previous interactions with $a$.

\section{Design of the Improvement Calculation FLS}

In ordered to calculate the necessary improvement of each criterion, the proposed model uses a fuzzy logic system. As with other FLSs, we need to define input parameters, the membership functions of fuzzy sets for inputs, as well as the fuzzy logic rule for the engine, which determines the behaviour of the system. It is common for membership functions to overlap to allow for a smooth transition between states (Muñoz et al., 2014). For each input of the proposed improvement calculation FLS, we define three fuzzy sets to allow for an acceptable granularity level in the input space without dramatically increasing the number of states aiming to reduce the size of control rules' set. We propose that input parameters can High $(\mathrm{H})$, Medium (M), or Low
(L), while output signals can be Very High (VH), High (H), Medium (M), Low (L) or Very Low (VL)

\section{Input Parameters}

For the proposed fussy logic system, we use two input parameters, namely the Percentage Of Satisfaction of the most recent transaction (POS) reported back from the trustor and the Improvement of the most recent transaction (Imp) such that:

- The Percentage Of Satisfaction of the most recent transaction (POS) reported back from the trustor. This parameter represents how successful the trustee could model the needs of the trustor. A transaction with a large value of POS means that the trustee met the needs of the trustor

- The Improvement of the most recent transaction (Imp). This parameter is used to highlight what level of improvement can lead to the POS. This helps the trustor decide which criteria need more improvement. For example, a high POS, even with low improvement indicates that the criteria are not important. The trustee can satisfy the trustor's needs without much emphasis on this particular criterion

\section{Fuzzification}

The real number of parameters described earlier need to be mapped into the corresponding fuzzy logic membership values of proper sets. Membership functions or the fuzzification operators map the real number input values into the corresponding fuzzy sets by evaluating the degree of membership for each input parameter to each possible fuzzy set for the system. A membership function's output is usually a degree of membership in the corresponding fuzzy set within the interval $[0,1]$. Commonly used types for membership functions include Piecewise linear, Trapezoidal, Triangular and Gaussian (Mendel, 1995).

In this study, we use Gaussian membership function to fuzzify the two input parameters; POS and improvement described in section 5.1.1, such that:

$\mu_{B_{j}^{k}}(v)=e^{-\left(\frac{\left(d_{j}^{k}-v\right)^{2}}{2\left(\sigma_{j}^{k}\right)^{2}}\right)}$

where, $\sigma_{j}^{k}$ and $d_{j}^{k}$ are standard deviation and the mean values for the corresponding Gaussian MF, respectively. In the fuzzy set $B_{j}^{k}(k=L, M, H$ and $j=1$, 2 ), $v$ is a member of the fuzzy set $k$ (e.g., $B_{j}^{k}$ is the fuzzy set of high percentage of recent improvement when $j=2$ and $k=H$ ).

The output fuzzy set has the following Gaussian MF: 
$\mu_{E^{k}}(v)=e^{-\left(\frac{\left(d_{j}^{k}-v\right)^{2}}{2\left(\sigma_{j}^{k}\right)^{2}}\right)}$

where standard deviation and mean values for the corresponding Gaussian MF are $\sigma_{j}^{k}$ and $d_{j}^{k}$, respectively.

In the fuzzy set $E^{k}(k=V H, H, M, L, V L) v$ is a member of the fuzzy set $k$.

\section{Inference Rules}

Another important component of the FLS is the inference engine. Here we define the inference rules for the engine. These rules set the relationship between inputs and output. The fuzzy logic system's inference engine recognizes which rules are activated and evaluates the fuzzy output values based on each rule (Mendel, 1995). For example; consider the following rule for the improvement FLS:

"If POS is HIGH and the Imp is LOW then the calculated improvement is LOW."

This rule intuitively states that if the reported percent of satisfaction is high and the last improvement is LOW, then the trustee is expected to be satisfied with a low level of improvement for this criterion.

To ensures that the inputs' membership degree is reflected in the output, we use the well known inference method, Mamdani min-max (Mamdani and Assilian, 1975), where the minimum of the maximum membership values of the intersections of the fuzzy value antecedent and input pairs determines the degree of membership of rule conclusions (Griffiths et al., 2006).

The output fuzzy set of the $j$ th rule is $I M P_{j}^{\prime}$ such that:

$$
\mu_{F I M P_{j}}^{\prime}(f t)=\mu_{P O S_{i}}(p o s) \wedge \mu_{I M P_{j}}(i m p)
$$

The aggregation of the fuzzy set's outputs due to rules with non-zero matching antecedent degree into a single fuzzy set is necessary before defuzzification. The overall fuzzy output from individual outputs is calculated by taking the maximum value where one or more terms overlap (Pham and Castellani, 2002).

In the improvement calculation FLS, the max function of the aggregation method processes the five truncated fuzzy sets coming from the nine rules to generate the fuzzy set of the outcome 'Improvement' before defuzzification:

$$
\mu_{I M P_{j}}^{\prime}(\text { imp })=\max _{1 \leq i \leq 9} \mu_{I M P_{i}}^{\prime}(\text { imp })
$$

Table 1 presents the proposed rules for the improvement calculation FLS.
Table 1: Improvement calculation fuzzy logic subsystem rules

\begin{tabular}{llll}
\hline Rule & POS & $\begin{array}{l}\text { Improvement of the } \\
\text { most recent transaction }\end{array}$ & Output \\
\hline 1 & L & L & VH \\
2 & M & L & H \\
3 & H & L & L \\
4 & L & M & H \\
5 & M & M & VH \\
6 & H & M & VL \\
7 & L & H & L \\
8 & M & H & M \\
9 & H & H & M \\
\hline
\end{tabular}

\section{Defuzzification}

Defuzzification is the last step of the fuzzy inference. During defuzzification, the fuzzy set of the outcome 'Improvement' resulting from the aggregation process mapped to a numerical value. Various defuzzification methods have been proposed in the literature. The centroid method is a well-known defuzzification method (Pappis and Siettos, 2005). It produces an output related to all fuzzy rules based on the center of gravity of the resulting fuzzy space. The center of gravity for the centroid method is given by:

$i m p^{\prime}=\frac{\int_{i m p} \mu_{I M P}^{\prime}(i m p)(i m p . d f t)}{\int_{i m p} \mu_{I M P}^{\prime}(i m p)(d f t)}$

where, $i m p^{\prime}$ is the numerical output and $\mu_{I M P}^{\prime}(i m p)$ is the membership function of the aggregated fuzzy set IMP.

Unlike RLTE (Aref and Tran, 2015b), ATeIF (Aref and Tran, 2017a) and ITE (Aref and Tran, 2020a), MCFTE does not depend on implicit feedback from trustors to tune up the performance of trustee(s). Arguably, a trustee can depend on the level of satisfaction reported by each trustor per criterion with its importance, combined with the utility gain proposed by the trustee for the same criterion to determine the necessary performance enhancement, if each trustor is will to accurately report the level of satisfaction per criterion. However, when trustors are willing to report only a general satisfaction value per transaction accurately, trustees need to be able to predict the appropriate value per criterion that satisfies the needs of each trustor.

MCFTE tune-up trustee(s) performance is based on explicit feedback from trustors on their level of satisfaction with recent transactions, where each trustor is willing to report only a general satisfaction value per transaction accurately. If a trustor $x$ reports a low level of satisfaction, the corresponding trustee needs to enhance its performance, particularly regarding important criteria. However, if a trustor reports a high level of satisfaction, the corresponding trustee can 
carefully reduce its performance, particularly concerning low importance criteria.

\section{Performance Analysis}

Existing trust establishment models (Aref and Tran, 2020b; 2017b; 2015b; 2016; 2015c; Tran et al., 2014; Burnett et al., 2011) have been analyzed using simulation to evaluate their effectiveness under different conditions, as obtaining comprehensive real-world data sets can be challenging. We are currently not aware of any agreed-upon benchmark that enables comparing various performance results, as simulation analysis differs among different works.

To get more insight on the performance of the proposed model, we compare it with the most relevant work that uses explicit feedback known as MCTE (Aref and Tran, 2017b), the most recent work that uses both implicit and explicit feedback known as ITE (Aref and Tran, 2020a) and the wildly compared with model; the RI (Burnett et al., 2011).

\section{Performance Measures}

It is argued in (Sen, 2013) that the cost of acquiring trust may be compensated if enhanced levels of trust can lead to further profitable interactions. Therefore building high level of trust can be advantageous for rational trustees. As with (Aref and Tran, 2020b; 2017b), we use the following measures in our simulation analysis:

- $\quad$ Level of Trust: We calculate the level of trust due to a particular model as the average direct trust for all agents (trustees) that use the trust establishment model. Even though exact level of trust depends highly dependent on the trust evaluation model used by trustors, the metric can be considered as indicator in situations where a general purpose trust evaluation model used by trustors

- Delivered utility gain: As an indicator of the efforts required by trustees to enhance their level of trust. When selecting interaction partners is based on trust, it is arguable that an honest trustee $b$ can achieve a higher level of trust and gain a larger portion of overall transactions if the trustee provides the highest possible utility gain for each transaction with each trustor. As this usually increases the cost of interacting with trustors, a rational trustee should aim to fulfill a higher number of transactions while providing a low utility gain as possible. In this simulation analysis, this metric is calculated as the sum of all delivered utility gain by the group of trustees empowered with the trust establishment model being analyzed, divided by the number of transactions performed by trustees who used that model:

$$
\text { Average }_{l}(s)=\frac{\sum_{b \in}^{q} \sum_{a \in A} \sum_{k=1}^{N T R_{b}^{a}} U_{a}^{b}(k)}{\sum_{b \in B} \sum_{a \in A} N T R_{b}^{a}}
$$

$$
\begin{aligned}
& \text { Where: } \\
& \begin{aligned}
l= & \text { The trust establishment model that trustee } \\
& b \text { used } \\
N T R_{b}^{a}= & \text { The number of transactions between } \\
& \text { trustor } a \text { and trustee } b
\end{aligned}
\end{aligned}
$$

\section{Simulation Environment}

In order to compare the performance of different trust establishment models, we used a scenario-simulator as described in (West et al., 2010) where scenarios are generated before simulation and not changed through the simulation phase. The process of generating scenario files encodes other parameters, including the number of agents in the MAS, the number of trustors, the number of trustees and the profile of each agent. Thus, the same scenario can be simulated using various trust establishment models. We aim to fix every aspect of a scenario run, such that we can relate the performance difference between runs with the trust establishment model used.

In this study, a scenario can be seen as a sequence of "interactions following requests for interactions", where each request determines the particular agent seeking the service (the trustor) and the service being requested. At simulation run-time, trustors select particular trustees for interaction based on trust evaluation model. From trustees perspective, the value for different dimensions of the response is determined at simulation time based on the trust establishment model.

We used MASON (Luke et al., 2005), a discreteevent simulation toolkit for MAS, in this performance analysis where trustors consume services and trustees provide them. In this study, the performance of each trustee in a particular service is assumed to be independent of that in any other service. Consequently, we assumed the existence of one service type in the simulated environment to reduce the complexity of simulation. The time value for interactions is calculated using simulation step, where each step is considered a single time unit and interactions that take place within the same time unit (simulation step) are considered simultaneous interactions. The system is not assumed to have a trust certification mechanism, trustors assumed to be able to request any number of trustees to bid and third-party witnesses are assumed to be honest. As locating other agents is not part of the proposed model, we assume that agents can locate each other through the system. Similarly, we did not consider network communication effects in this simulation and assumed that agent are reachable by other agents in the system. 
Trustors assumed to use a simple probabilistic trust evaluation model as follows:

$$
\text { trust }_{b}^{a}=0.5 * \text { indirect Trust }_{b}^{a}+0.5 * \text { directTrust }_{b}^{a}
$$

where, indirect trust represents the reputation of a trustee in the community and direct trust, is the average satisfaction from interactions performed with the trustee.

Initially, trustors assume a neutral trust level for every trustee. Initially trust level is set to:

trust $_{b}^{a}=\frac{\max \text { Trust }+\min \text { Trust }}{2}$

After selecting a trustee $b$, trustor $a$ interacts with $b$ and gains utility in the same simulation step. Any trustee is capable of serving many trustors simultaneously, where as the frequency at which a trustor request a service depends on it level of activity. After each interaction, $a$ updates the level of direct trust of its interaction partner.

In this simulation analysis, values plotted on charts are calculated as the average value for ten randomly generated scenarios.

As we compare the proposed model with ITE (Aref and Tran, 2020a), MCTE (Aref and Tran, 2017b) and RI (Burnett et al., 2011); we assume that all trustees are honest and the only difference is the trust establishment model used.

Table 2 presents the number of agents and other parameters used in the proposed model and those applied in the environment for base cases. When testing the effect of a particular parameter, other parameters are set to those base values.

\section{Experimental Results}

\section{Demand Level Effects}

As each trustor's needs for the same service may vary in various contexts, we need to study the impacts of demand level on the behavior of MCFTE. For example, in the context of a e-commerce, various buyers require various levels of "fast delivery". Therefor, in this study, we analyze three extreme demand cases where all trustors belong to the same category in each case. We consider the case of trustors low demand (i.e., easygoing), the case of trustors with high demand (demanding) and the case of trustors with intermediate (normal) demand. Easy-going trustors require less any level of service between 0 and $35 \%$ of the maximum possible utility gain for each criterion, whereas demanding trustors require any service level between 65 and $100 \%$ of the maximum possible utility gain for each criterion normal trustors require something in between. Simulation results of the models in terms of average trust and utility gain under different demand levels are presented in Fig. 3 and 4, showing that MCFTE empowered trustees have a high average trust compared to MCTE and higher average trust to ITE and RI (Fig. 3, while providing a utility comparable to MCTE, but higher than the other two models (Fig. 4a and 4b) for normal and demanding trustors and considerably lower than the utility gain provided by MCTE (Fig. 4c for easy-going trustors, which is comparable to ITE and slightly higher than RI. This is because MCFTE relies on explicit feedback and attempts to focus on which criteria to improve to achieve partners' satisfaction. Figure 3c shows that all models cause trustees to stay trustworthy while providing low utility gain for easy-going trustors. Nevertheless, RI achieved the least level of (about 80\%). While the proposed model attempts to start making a profit as the level of satisfaction is high enough, providing various utility levels according to importance level helps trustees achieve a relatively high level of trust at a reasonable cost in terms of provided utility gain.

\section{Activity Level Effects}

While some trustors might request a service frequently, others may request the service less frequently or even rarely. Therefore, in this study, we analyze three extreme cases for the trustors level of activity, where all trustors belong to the same category in each case. We consider the case of low activity trustors (i.e., rarely active), the case of highly active trustors (frequently) and the case of trustors with intermediate (normal) activity level.

Rarely active trustors request a particular service with a probability between 0 and $35 \%$ in each simulation step, frequently active trustors request a particular service with a probability between 65 and $100 \%$ and normal trustors request a particular service with probability in between the other two categories.

Table 2: Used parameters

\begin{tabular}{ll}
\hline Parameter & Value \\
\hline Number of trustees & 12 \\
Number of trustors & 88 \\
Number of agents & 100 \\
Percentage of highly active trustors & $30 \%$ \\
Percentage of regular active trustors & $40 \%$ \\
Percentage of low active trustors & $30 \%$ \\
Percentage of high demand trustors & $30 \%$ \\
Percentage of low demand trustors & $30 \%$ \\
Percentage of regular demand trustors & $40 \%$ \\
Number of simulation steps & 1000 \\
Number of criteria & 10 \\
$\operatorname{Min}^{b}\left(t_{d_{i}}\right)$ & 0.1 \\
$\operatorname{Max}^{b}\left(t_{d_{i}}\right)$ & 1 \\
\hline
\end{tabular}




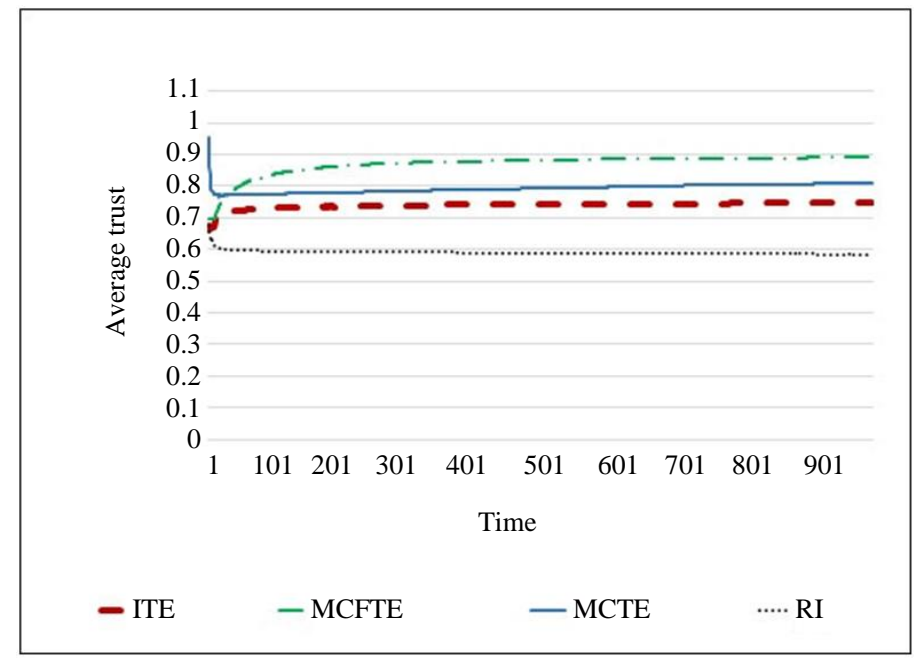

(a)

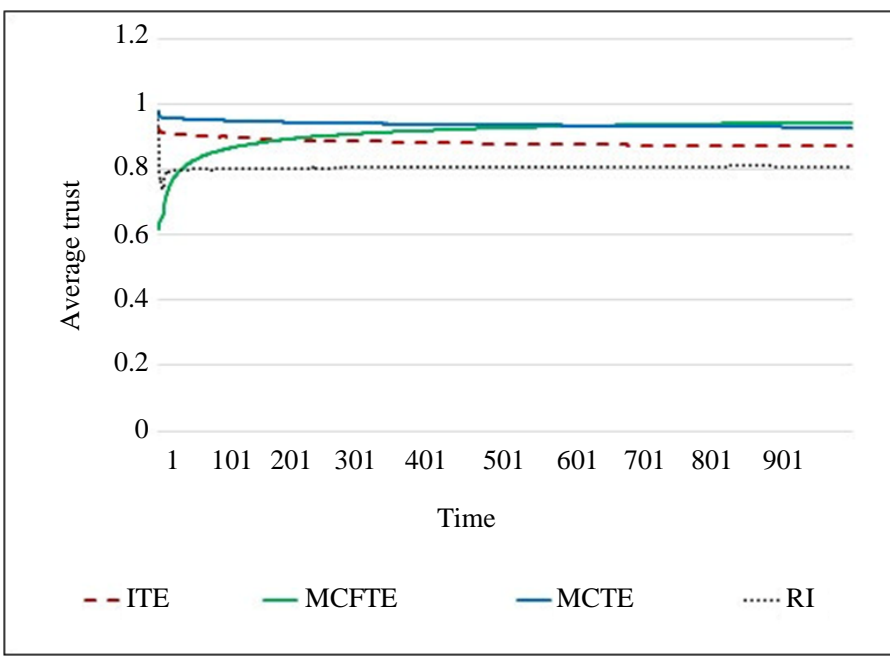

(b)

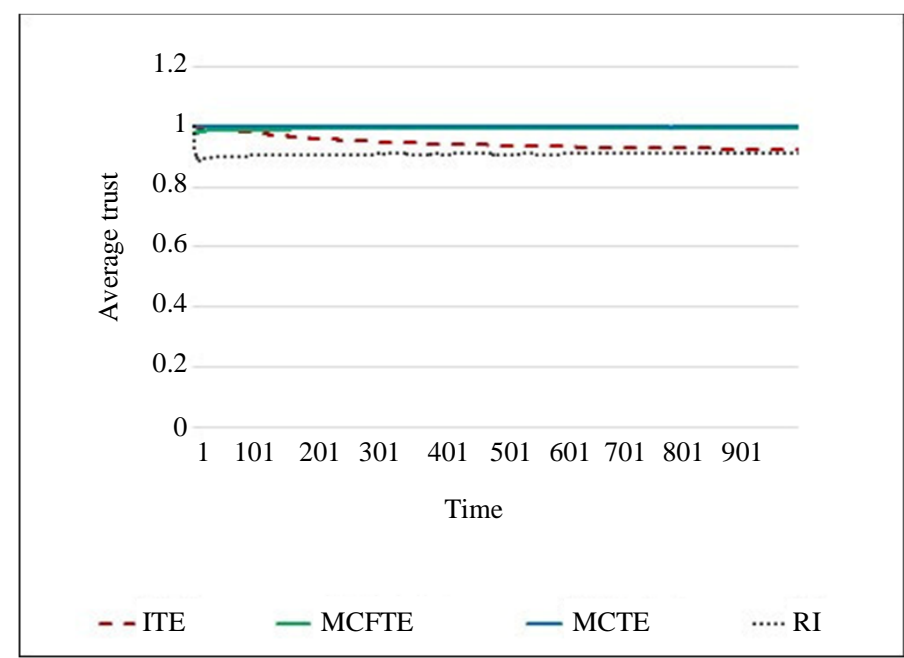

(c)

Fig. 3: Effect of trustors' demand on average trust (a) high demanding trustors; (b) regular demanding trustors; (c) low demanding trustors 


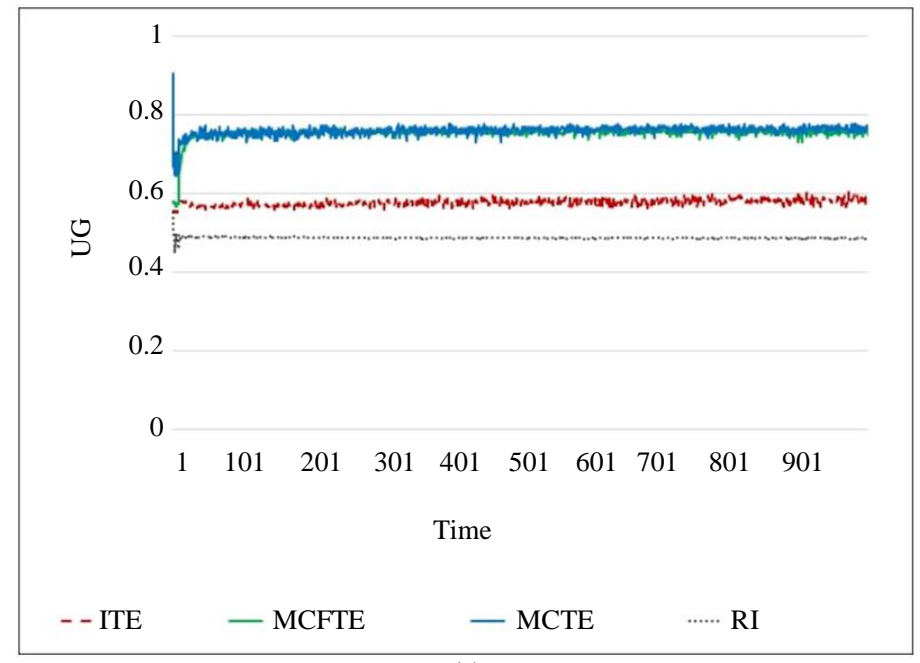

(a)

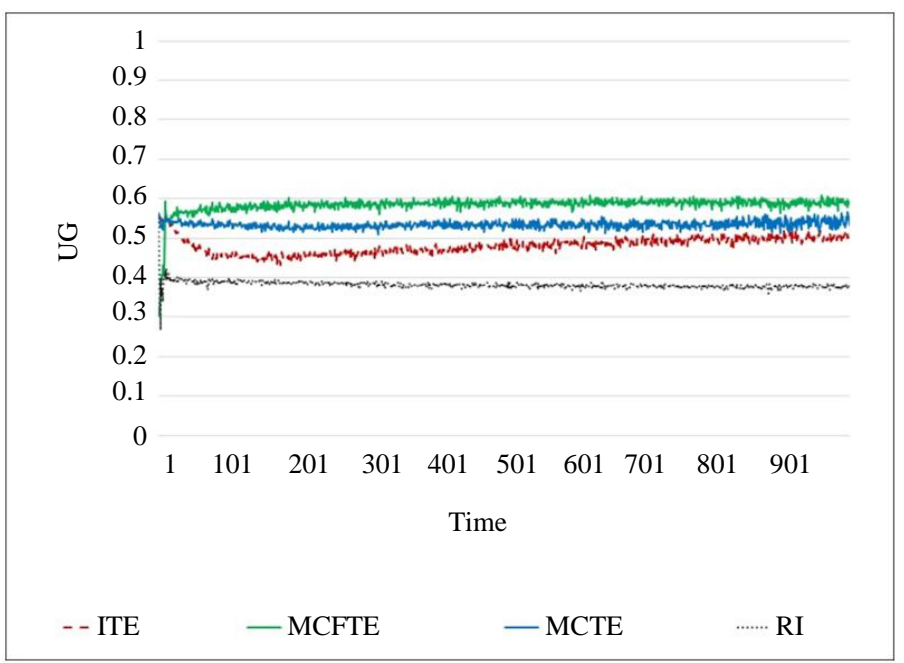

(b)

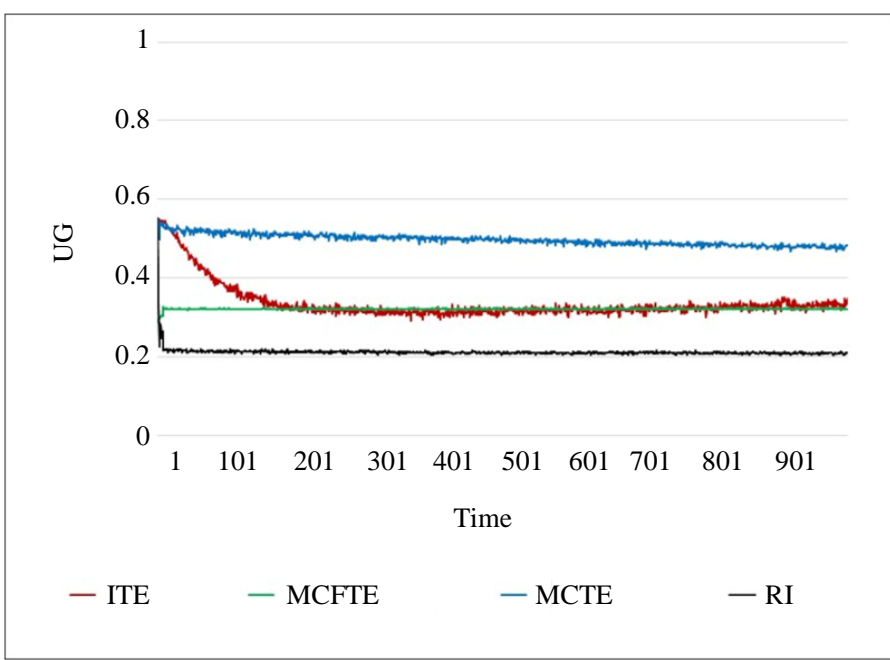

(c)

Fig. 4: Effect of trustors' demand on utility gain (a) highly demanding trustors; (b) regular demanding trustors; (c) low demanding trustors 


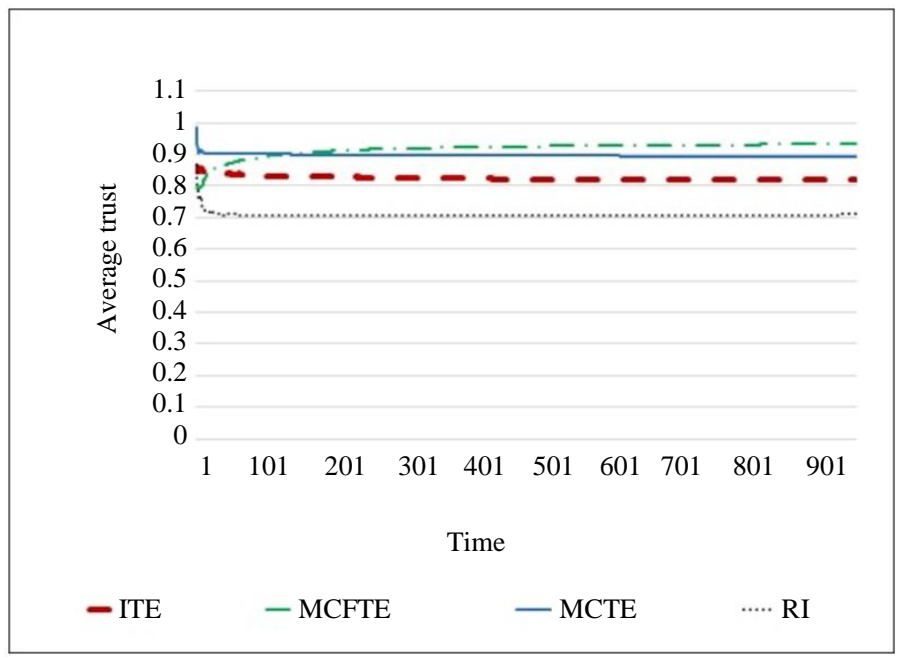

(a)

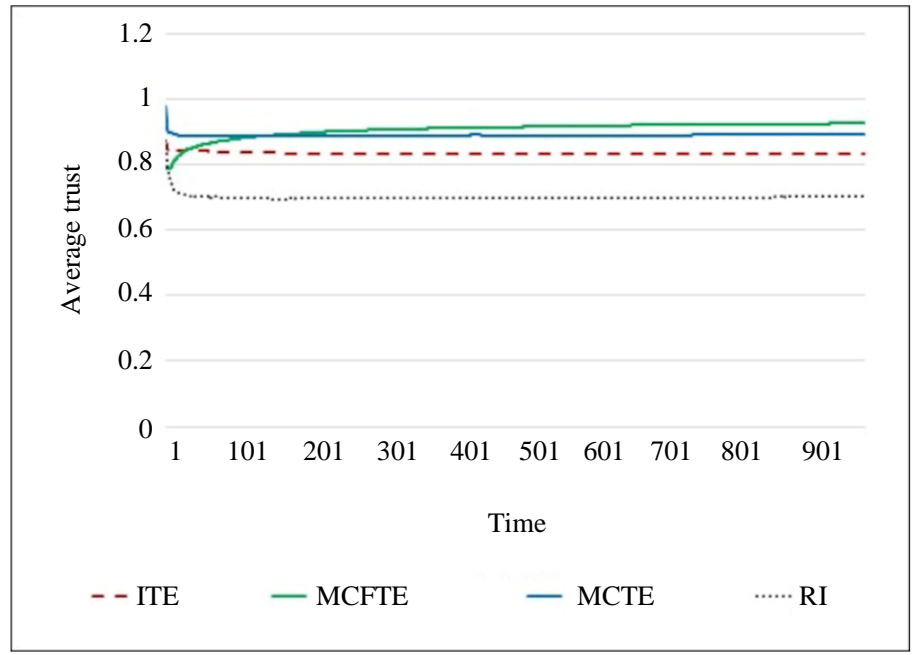

(b)

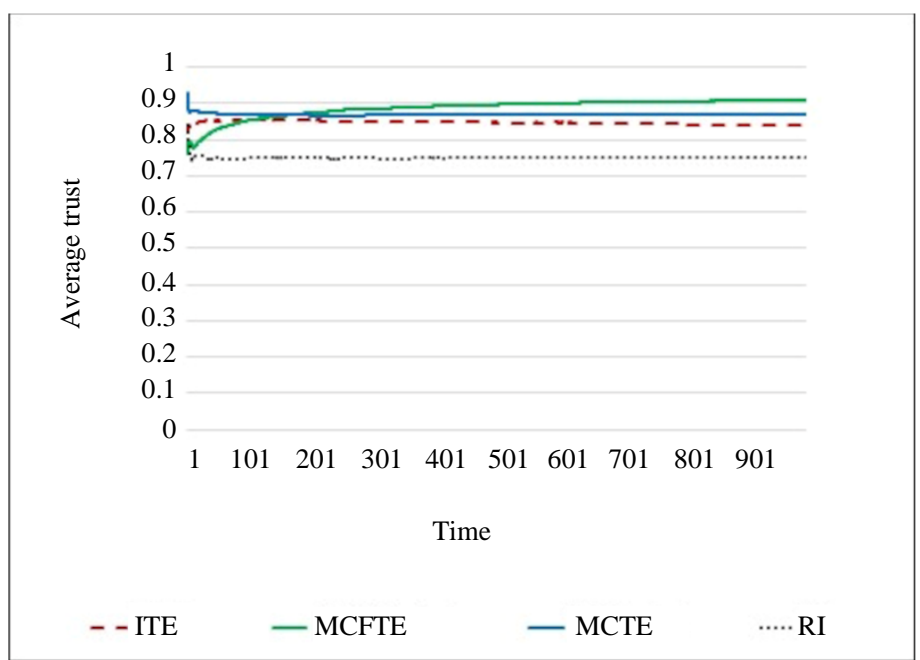

(c)

Fig. 5: Effect of trustors' activity on average trust (a) high activity trustors; (b) regular activity trustors; (c) low activity trustors 


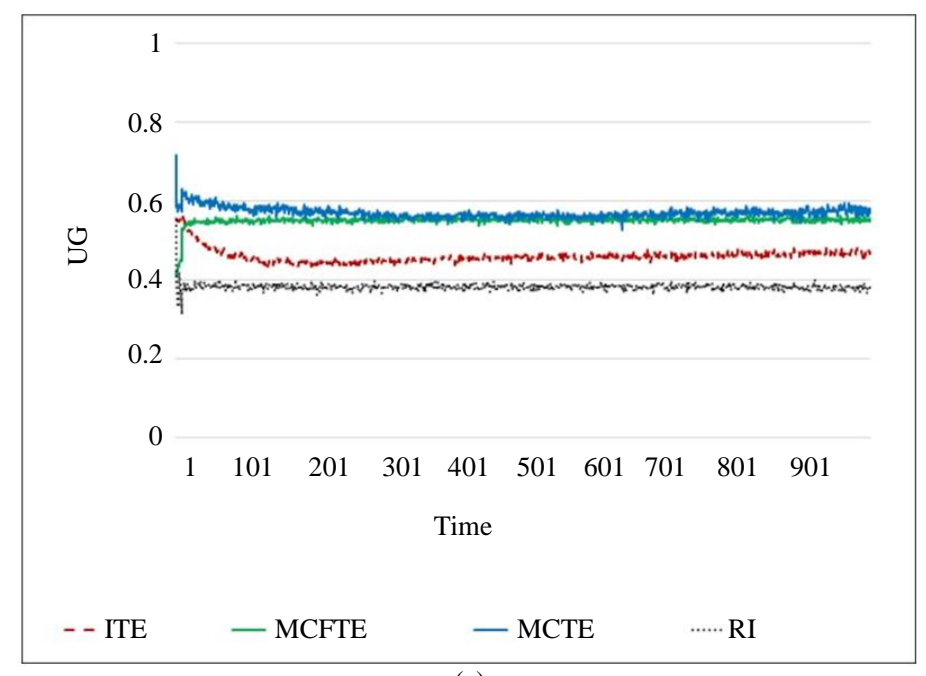

(a)

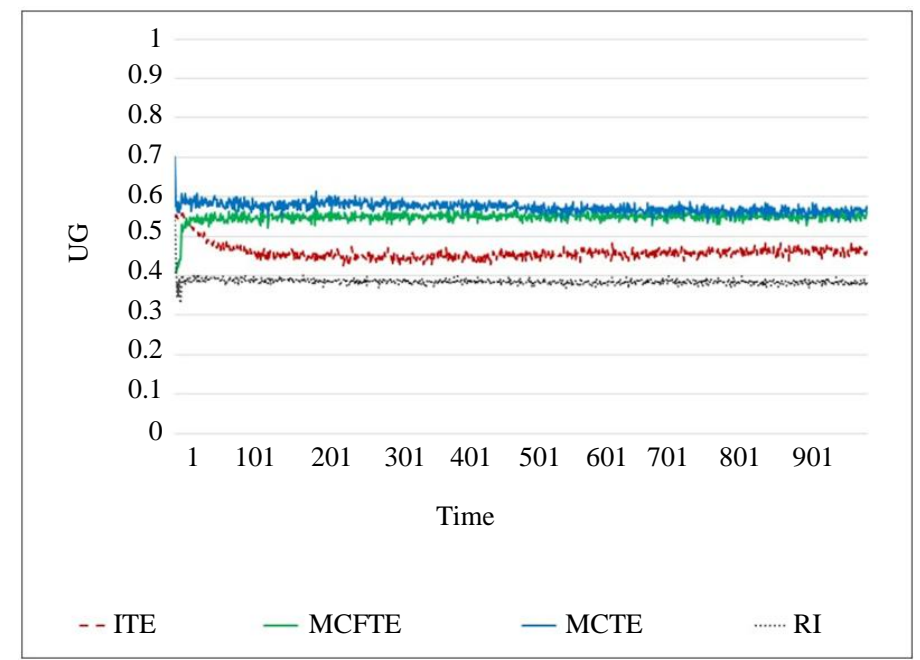

(b)

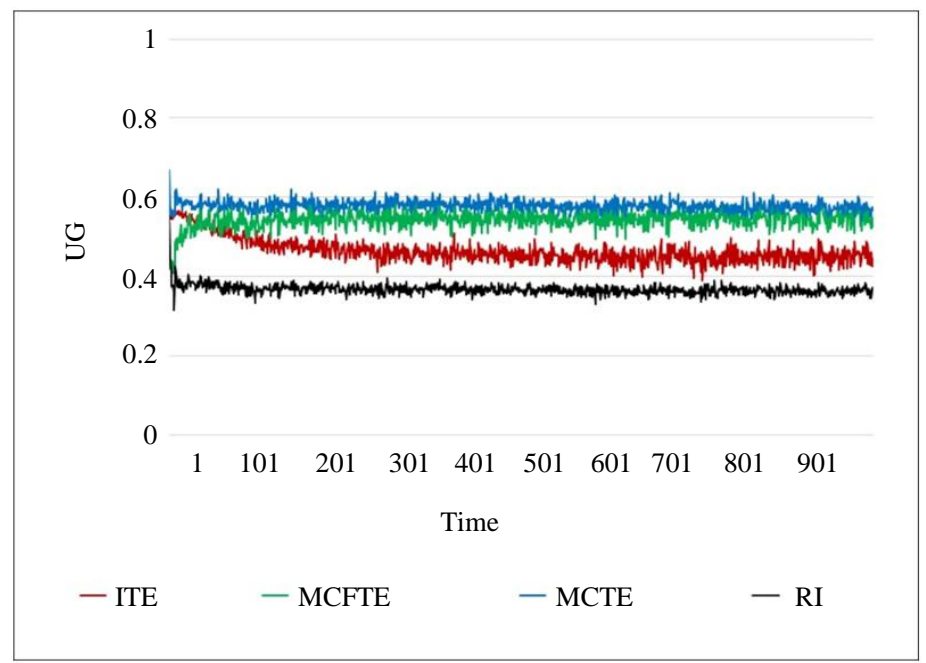

(c)

Fig. 6: Effect of trustors' activity on utility gain (a) high activity trustors; (b) regular activity trustors; (c) low activity trustors 
Simulation results of the models in terms of average trust and utility gain under different demand levels are presented in Fig. 5 and 6 which do not show significant difference for the level of trust for the models under various levels of activity analyzed; specifically models that do not relay on retention, namely: MCTE and MCFTE. On the other hand, those models seem to provide the highest utility gain compared to other models. The proposed model can achieve a level of trust comparable to ITE and MCTE for different activity levels analyzed. As ITE depends on both explicit and implicit feedback, it seems to be able to avoid providing extra high utility gain as the level of activity decreases.

\section{Conclusion and Future Work}

This study presented a multi-criteria, distributed model for trust establishment in MASs based on fuzzy logic referred to as MCFTE. The proposed model targets situations where trustors select trustees to interact with based on trust evaluation when trustors are willing to make available for use feedback on interactions in the form of a single satisfaction value per multi-criterion interaction. The model attempts to predict the necessary improvement per criterion. As rational trustees aim to enhance their level of trust to increase their potential to be chosen for interactions later in the future, the proposed model attempts to tune up trustee(s) performance to enhance their level of trust based on explicit feedback from trustors. The simulation analysis results indicate that MCFTE can help trustees achieve higher trust levels and get better chances to be selected as partners for interactions in MASs when trustors select trustees based on trust.

We want to extend the MCFTE to consider implicit feedback in addition to explicit feedback and address social relationships between agents. Also, we would like to analyze the impact of more parameters on performance of the model, trustees' capacity, partner selection mechanisms and the trust evaluation mechanism used by trustors. Determining values for the model's parameters dynamically and comparing the performance of MCFTE with other related trust establishment models is left as future work.

\section{Author's Contributions}

Abdullah Aref: Participated in the creation of the model, carried out the experimental studies and participated in drafting the script.

Eman Omar: Participated in the creation of the model and participated in drafting the script.

All authors read and approved the final manuscript.

\section{Ethics}

All information provided in this study is confidential and unique. This paper has neither been published nor is under review elsewhere. There are no ethical issues associated with this research.

\section{References}

Aref, A., \& Tran, T. (2014, September). Using fuzzy logic and Q-learning for trust modeling in multi-agent systems. In 2014 Federated Conference on Computer Science and Information Systems (pp. 59-66). IEEE.

Aref, A., \& Tran, T. (2015a, December). FTE: A fuzzy logic based trust establishment model for intelligent agents. In 2015 IEEE/WIC/ACM International Conference on Web Intelligence and Intelligent Agent Technology (WI-IAT) (Vol. 2, pp. 133-138). IEEE.

Aref, A., \& Tran, T. (2015b, August). RLTE: A reinforcement learning based trust establishment model. In 2015 IEEE Trustcom/BigDataSE/ISPA (Vol. 1, pp. 694-701). IEEE.

Aref, A., \& Tran, T. T. (2015c, January). A trust establishment model in multi-agent systems. In AAAI Workshop: Incentive and Trust in E-communities.

Aref, A., \& Tran, T. T. (2016, January). Modeling trust evaluating agents: towards a comprehensive trust management for multi-agent systems. In AAAI Workshop: Incentives and Trust in Electronic Communities.

Aref, A., \& Tran, T. (2017a, May). Acting as a Trustee for Internet of Agents in the Absence of Explicit Feedback. In International Conference on ETechnologies (pp. 3-23). Springer, Cham.

Aref, A., \& Tran, T. (2017b). Multi-criteria trust establishment for Internet of Agents in smart grids. Multiagent and Grid Systems, 13(3), 287-309.

Aref, A., \& Tran, T. (2018). A hybrid trust model using reinforcement learning and fuzzy logic. Computational Intelligence, 34(2), 515-541.

Aref, A., \& Tran, T. (2020a). An integrated trust establishment model for the internet of agents. Knowledge and Information Systems, 62(1), 79-105.

Aref, A., \& Tran, T. (2020b). An integrated trust establishment model for the internet of agents. Knowledge and Information Systems, 62(1), 79-105.

Burnett, C. (2011). Trust assessment and decisionmaking in dynamic multi-agent systems (Doctoral dissertation, University of Aberdeen, UK).

Burnett, C., Norman, T. J., \& Sycara, K. (2011). Decisionmaking with trust and control in multi-agent systems. In Twenty Second International Joint Conference on Artificial Intelligence (Vol. 10, pp. 241-248).

Georgoulas, S., Moessner, K., Mansour, A., Pissarides, M., \& Spapis, P. (2012, June). A fuzzy reinforcement learning approach for pre-congestion notification based admission control. In IFIP International Conference on Autonomous Infrastructure, Management and Security (pp. 26-37). Springer, Berlin, Heidelberg. 
Granatyr, J., Botelho, V., Lessing, O. R., Scalabrin, E. E., Barthès, J. P., \& Enembreck, F. (2015). Trust and reputation models for multiagent systems. ACM Computing Surveys (CSUR), 48(2), 1-42.

Griffiths, N., Chao, K. M., \& Younas, M. (2006, July). Fuzzy trust for peer-to-peer systems. In 26th IEEE International Conference on Distributed Computing Systems Workshops (ICDCSW'06) (pp. 73-73). IEEE.

Huynh, T. D., Jennings, N. R., \& Shadbolt, N. R. (2006). An integrated trust and reputation model for open multi-agent systems. Autonomous Agents and Multi-Agent Systems, 13(2), 119-154.

Josang, A., \& Ismail, R. (2002, June). The beta reputation system. In Proceedings of the 15 th bled electronic commerce conference (Vol. 5, pp. 2502-2511).

Luke, S., Cioffi-Revilla, C., Panait, L., Sullivan, K., \& Balan, G. (2005). Mason: A multiagent simulation environment. Simulation, 81(7), 517-527.

Malik, Z., \& Bouguettaya, A. (2009). Trust Management for Service-Oriented Environments. Springer Science \& Business Media.

Mamdani, E. H., \& Assilian, S. (1975). An experiment in linguistic synthesis with a fuzzy logic controller. International journal of man-machine studies, 7(1), $1-13$.

Mendel, J. M. (1995). Fuzzy logic systems for engineering: A tutorial. Proceedings of the IEEE, 83(3), 345-377.

Moradi, M. H., Razini, S., \& Hosseinian, S. M. (2016). State of art of multiagent systems in power engineering: A review. Renewable and Sustainable Energy Reviews, 58, 814-824.

Muñoz, P., Laselva, D., Barco, R., \& Mogensen, P. (2014). Dynamic traffic steering based on fuzzy QLearning approach in a multi-RAT multi-layer wireless network. Computer Networks, 71, 100-116.

Pappis, C. P., \& Siettos, C. I. (2005). Search Methodologies. Fuzzy Reasoning, 437-474.

Pham, D. T., \& Castellani, M. (2002). Action aggregation and defuzzification in Mamdani-type fuzzy systems. Proceedings of the Institution of Mechanical Engineers, Part C: Journal of Mechanical Engineering Science, 216(7), 747-759.

Pinyol, I., \& Sabater-Mir, J. (2013). Computational trust and reputation models for open multi-agent systems: a review. Artificial Intelligence Review, 40(1), 1-25.

Raju, G. V. S., Zhou, J., \& Kisner, R. A. (1991). Hierarchical fuzzy control. International journal of control, 54(5), 1201-1216.

Ramchurn, S. D., Huynh, D., \& Jennings, N. R. (2004). Trust in multi-agent systems. The Knowledge Engineering Review, 19(1), 1-25.
Ross, T. J. (2010). Fuzzy Logic with Engineering Applications. John Wiley \& Sons, Ltd.

Saini, R., \& Gautam, R. K. (2011, October). Establishment of dynamic trust among nodes in mobile ad-hoc network. In 2011 International Conference on Computational Intelligence and Communication Networks (pp. 346-349). IEEE.

Supriya, M., Sangeeta, K., \& Patra, G. K. (2016). A Fuzzy based Hierarchical Trust Framework to rate the Cloud Service Providers based on Infrastructure Facilities. International Journal of Performability Engineering, 12(1).

Sen, S. (2013, May). A comprehensive approach to trust management. In Proceedings of the 2013 international conference on Autonomous agents and multi-agent systems (pp. 797-800).

Shen, Y. C., Zhang, H., \& Duan, X. L. (2015). Trust model of P2P network based on fuzzy degree of credit. In Applied Mechanics and Materials (Vol. 738, pp. 1231-1235). Trans Tech Publications Ltd.

Shirgahi, H., Mohsenzadeh, M., \& Haj Seyyed Javadi, H. (2017). A three level fuzzy system for evaluating the trust of single web services. Journal of Intelligent \& Fuzzy Systems, 32(1), 589-611.

Singh, S., \& Sidhu, J. (2016a). An approach for determining trustworthiness of individuals in a webbased social network. Arabian Journal for Science and Engineering, 41(2), 461-477.

Singh, S., \& Sidhu, J. (2016b). An approach for determining trustworthiness of individuals in a webbased social network. Arabian Journal for Science and Engineering, 41(2), 461-477.

Tran, T., \& Cohen, R. (2004, July). Improving user satisfaction in agent-based electronic marketplaces by reputation modelling and adjustable product quality. In Proceedings of the Third International Joint Conference on Autonomous Agents and Multiagent Systems-Volume 2 (pp. 828-835).

Tran, T. T., Cohen, R., Langlois, E., \& Kates, P. (2014). Establishing Trust in Multiagent Environments: Realizing the Comprehensive Trust Management Dream.TRUST@AAMAS, 1740, 35-43.

West, A. G., Kannan, S., Lee, I., \& Sokolsky, O. (2010). An evaluation framework for reputation management systems. In Trust Modeling and Management in Digital Environments: From Social Concept to System Development (pp. 282-308). IGI Global.

$\mathrm{Yu}$, H. (2014). Situation-aware trust management in multi-agent systems (Doctoral dissertation).

Yu, H., Miao, C., An, B., Leung, C., \& Lesser, V. R. (2013a, August). A Reputation Management Approach for Resource Constrained Trustee Agents. In IJCAI (Vol. 13, pp. 418-424). 
Yu, H., Shen, Z., Leung, C., Miao, C., \& Lesser, V. R. (2013b). A survey of multi-agent trust management systems. IEEE Access, 1, 35-50.

Yu, H., Shen, Z., Miao, C., \& An, B. (2013c, May). A reputation-aware decision-making approach for improving the efficiency of crowdsourcing systems. In AAMAS (Vol. 13, pp. 1315-1316).
Zhang, J., \& Cohen, R. (2007, July). Design of a mechanism for promoting honesty in emarketplaces. In PROCEEDINGS OF THE NATIONAL CONFERENCE ON ARTIFICIAL INTELLIGENCE (Vol. 22, No. 2, p. 1495). Menlo Park, CA; Cambridge, MA; London; AAAI Press; MIT Press; 1999. 